\title{
METODOLOGIA PARA VALIDAÇÃO DE DADOS TRMM PARA UBERABA (MG)
}

\author{
METHODOLOGY FOR TRMM DATA VALIDATION FOR \\ UBERABA (MG) \\ METODOLOGÍA PARA VALIDACIÓN DE DATOS TRMM PARA \\ $U B E R A B A(M G)$
}

\author{
Sérgio Roberto Araújo \\ Acadêmico do curso de Geografia \\ Universidade Federal do Triângulo Mineiro \\ serginho.uberaba@gmail.com
}

Fabio de Oliveira Sanches

Prof. Adjunto na Universidade Federal do Triângulo Mineiro

Laboratório de Geomorfologia, Clima e Solos - LAGECS

fsanches.73@gmail.com

Ricardo Vicente Ferreira

Prof. Adjunto na Universidade Federal do Triângulo Mineiro Laboratório de Cartografia e Geoprocessamento - CARTOGEO

rcrdvf@gmail.com

Resumo: Estimativas de chuvas feitas por satélites têm contribuído para ampliar a cobertura de dados climatológicos em países de dimensóes continentais como o Brasil. No entanto, a precisão de seus dados ainda é questionável quando comparados aos dados coletados em superfície. O objetivo do trabalho foi avaliar a qualidade dos dados estimados pelo satélite TRMM para Uberaba (MG) a partir de dados observados em superfície. A aplicaçáo da estatística descritiva (Média, Mediana, Amplitude, Desvio Padrão e Coeficiente de Variaçáo) e análise Box Plot em ambos os conjuntos de dados demonstrou forte similaridade. A Correlação de Pearson demonstrou que ambos os conjuntos de dados apresentam elevado nível de similaridade $\left(\mathrm{R}^{2}=0,994\right)$. O Teste $t$ de Student demonstrou que ambos os conjuntos de dados são, estatisticamente, homogêneos. Sendo assim, os resultados demonstraram excelente qualidade dos dados estimados pelo TRMM para Uberaba (MG) quando comparados aos dados observados.

Palavras-chave: Precipitações, testes estatísticos, box plot, Triângulo Mineiro, satélites. 


\begin{abstract}
Rainfall estimates made by satellites have contributed to the coverage of climatological data in continental countries such as Brazil. However, its accuracy is still questionable when compared to the data collected on the surface. The objective was to evaluate the quality of the data estimated by the TRMM satellite to Uberaba (MG) from data observed on surface. Descriptive statistics (Average, Median, Amplitude, Standard Deviation and Coefficient of Variation) and box plot analysis showed strong similarity. The Pearson correlation showed that the data sets have a hig h level of similarity $\left(\mathrm{R}^{2}=0.994\right)$. The Student t-test showed that the data sets are statistically homogeneous. Thus, the results showed excellent data quality estimated by TRMM to Uberaba (MG) when compared to the observed data.
\end{abstract}

Keywords: Rainfall, statistical tests, box plot, Triângulo Mineiro, Satellites.

Resumen: Estimaciones de lluvias realizadas por los satélites han ayudado a ampliar la cobertura de los datos climatológicos en los países de dimensiones continentales como Brasil. Sin embargo, suprecisión es todavía cuestionable si se compara con los datos recogidos en la superficie. El objetivo fue evaluar la calidad de los datos por el satélite TRMM estimado a Uberaba (MG) a partir de la fecha observada en la superficie. La estadística descriptiva (media, mediana, rango, desviación estándar y coeficiente de variación) y el análisis de diagrama de caja mostró una fuerte similitud. La correlación de Pearson mostró que ambos conjuntos de datos tienen un alto nivel de similitud $\left(\mathrm{R}^{2}=0,994\right)$. La prueba $\mathrm{t}$ de Student mostró que los datos son estadísticamente homogéneos. Por lo tanto, los resultados mostraron una excelente calidad de los datos estimados por TRMM a Uberaba (MG) en comparación con los datos observados.

Palabras clave: lluvias, pruebas estadísticas, gráfico de caja, Triângulo Mineiro, satélites.

\title{
INTRODUÇÃO
}

Uma das principais dificuldades encontradas para estudos climáticos, sobretudo em um país de proporçóes continentais como o Brasil, está na existência (ou não) de um conjunto de dados e que apresente significativa qualidade (SANCHES, 2015).

No Brasil, a instituição responsável pela coleta e disponibilidade de dados meteorológicos é o Instituto Nacional de Meteorologia (INMET), no entanto, o estado possui ainda outros organismos e agências que coletam e disponibilizam seus próprios conjuntos de dados.

O INMET dispóe, por meio de seu Banco de Dados Meteorológico para Ensino e Pesquisa (BDMEP) ${ }^{1}$ dados meteorológicos diários (pluviosidade, temperatura, umidade do ar, pressão atmosférica, insolação, velocidade e direção do vento) da sua rede de Estaçóes Meteorológicas Convencionais, com informaçôes a partir de janeiro de 1961.

A Agência Nacional de Águas (ANA) disponibiliza dados pluviométricos diários

Disponível em: <http://www.inmet.gov.br/portal/index.php?r=bdmep/bdmep> Acesso em: 
de diversas estações sob sua responsabilidade, por meio de sua plataforma hidroweb Sistema de Informações Hidrológicas. ${ }^{2}$ Entretanto, em ambas as fontes, os dados das diversas estaçôes pluviométricas e das Estações Meteorológicas Convencionais apresentam falhas passíveis de correção por meio de recursos estatísticos.

Da Silva et al. (2013), acreditam que as estimativas de precipitação por satélites vêm contribuindo para aumentar a cobertura de dados climatológicos. Dados de precipitaçáo estimados pelo satélite Tropical Rainfall Measuring Mission (TRMM) podem ser utilizados na ausência de dados das estaçóes meteorológicas convencionais de superfície.

Em órbita desde o final de 1997, o satélite TRMM foi desenvolvido em parceria entre a National Aeronautics and Space Administration (NASA) e Agência Japonesa de Exploração Aeroespacial (JAXA) para monitorar as precipitações nas áreas tropicais (COLLISCHONN et al., 2007).

Instalados a bordo do TRMM estão imageador de microondas (TMI), radar de precipitação (PR), radiômetro no visível e no infravermelho (VRS), sensor de energia radiante da superfície terrestre e das nuvens (CERES), e sensor imageamento de relâmpagos (LIS), sendo o radar de precipitação (PR). Sua órbita baixa (cerca de $403 \mathrm{~km}$ ) e não heliosíncrona permite altas resoluçóes espacial e temporal, com revisita estimada a cada três horas (NOBREGA et al., 2008; ANJOS et al., 2016).

Para Da Silva et al. (2013), as estimativas espaciais de precipitação constituem uma ferramenta extremamente útil, embora pouco precisas quando comparadas com dados pontuais medidas em superfície, tendo em vista que a precipitação é a variável que apresenta maior variabilidade espacial.

Collischonn et al. (2007), realizaram estudos na bacia do Paraguai Superior, onde analisaram e compararam os dados de campo em relação aos dados obtidos pelo satélite TRMM, e consideraram os resultados obtidos satisfatórios, mostrando que as estimativas deste satélite podem ser uma fonte alternativa de dados em escassez de informação.

A obtenção de dados climáticos por meio de satélites vem sendo utilizada com mais frequência nas últimas três décadas, com o desenvolvimento e aprimoramento de satélites ambientais, aumentando a quantidade de informações disponíveis, incluindo estimativas de precipitação (COLLISCHON, 2006; NÓBREGA et al., 2008; ANJOS et al., 2016).

Dessa forma, o objetivo desse trabalho é demonstrar uma metodologia para avaliar a qualidade dos dados estimados pelo satélite TRMM para Uberaba (MG) a partir dos dados observados em superfície.

\section{MATERIAL E MÉTODOS}

Para esse trabalho foram utilizados dados pluviométricos mensais estimados pelo satélite TRMM obtidos a partir do Laboratório de Sensoriamento Remoto Aplicado à

<http://www.snirh.gov.br/hidroweb> Acesso em: 
Agricultura e Floresta (LAF) da Divisão de Sensoriamento Remoto do Instituto Nacional de Pesquisas Espaciais (DSR-INPE) (https://www.dsr.inpe.br/laf/series/index.php).

Os dados do satélite TRMM foram estimados para a área da Estação Meteorológica de Superfície, em Uberaba, para o período de 2000 a 2012 (Figura 1b). Também foram utilizados dados pluviométricos mensais obtidos por meio do BDMEP para a Estação Meteorológica de Superfície de Uberaba (OMM 83577) (Figura 1a) no mesmo período.

\section{Figura 1 - Localização da Estação Meteorológica de Superfície de Uberaba (a) e ponto correspondente a coleta de dados do satélite TRMM (b)}

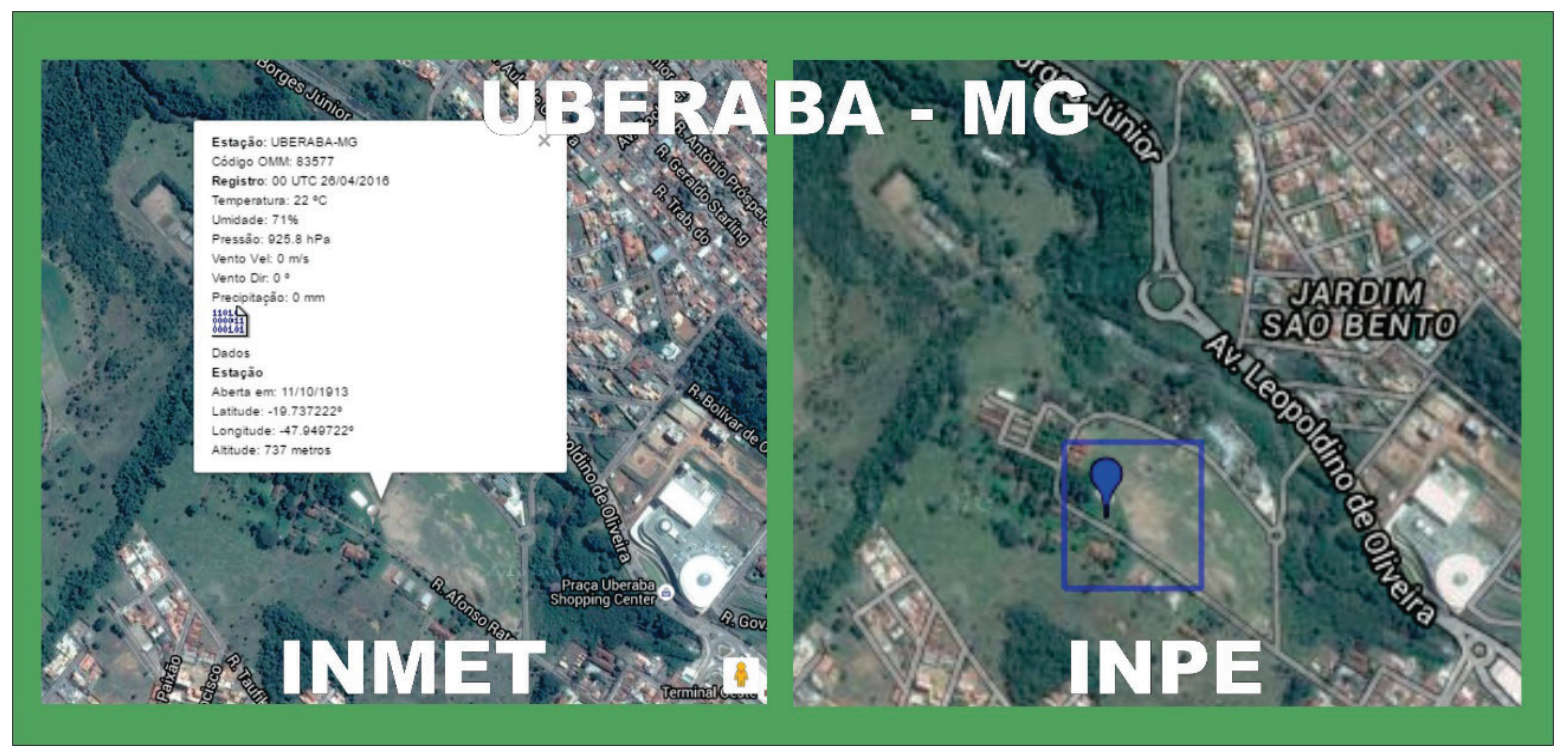

(a)

(b)

Fonte: Figura adaptada com informações dos sites do Instituto Nacional de Meterologia (www.inmet.gov.br) e do Instituto Nacional de Pesquisas Espaciais (www.inpe.br)

A organização de ambos os conjuntos de dados em totais mensais permitiu a aplicação da estatística descritiva (Média, Mediana, Amplitude, Desvio Padrão e Coeficiente de Variação (\%)). Em seguida, os dados foram analisados por meio da técnica Box Plot para avaliar a dispersáo entre os dados observados e os estimados pelo TRMM.

A etapa seguinte consistiu em se verificar a homogeneidade dos dados por meio da aplicação de Correlação de Pearson e da aplicação do Teste $t$ de Student (SPIGEL, 1993).

$\mathrm{Na}$ aplicação do Teste $t$ de Student considera-se que não existem diferenças entre as duas séries de dados $\left(\mu_{1}=\mu_{2}\right.$ e $\left.\sigma_{1}=\sigma_{2}\right)$, adotando-se, assim, a hipótese nula $\left(H_{0}\right)$. No entanto, se os resultados se mostrarem diferentes do que os esperados por essa hipótese $\left(H_{0}\right)$, conclui-se que as diferenças observadas são, estatisticamente, significativas.

O nível de significância adotado foi de 0,05 , ou seja, $5 \%$ de erro do valor que deveria ser aceito, proporcionando uma confiança de $95 \%$ de que a hipótese seja verdadeira. 
Entretanto, se essa hipótese $\left(H_{0}\right)$ for rejeitada quando deveria ser aceita, adotamos que foi cometido um erro do Tipo I (rejeição da hipótese nula como verdadeira).

Sendo assim, para se aceitar a hipótese $\left(H_{0}\right)$ como verdadeira, o escore $(\mathrm{z})$ de uma estatística amostral deve ser $-1,96 \leq \mathrm{z} \leq 1,96$.

Assim, no Teste $t$ de Student, temos:

$$
t=\frac{\mu_{1}-\mu_{2}}{\sigma \sqrt{1 / N_{1}+1 / N_{2}}}
$$

onde

$$
\sigma=\sqrt{\frac{N_{1} s_{1}^{2}+N_{2} s_{2}^{2}}{N_{1}+N_{2}-2}}
$$

e

$$
s^{2}=\frac{\left(\sum_{i=1}^{n} X_{i}-\mu\right)^{2}}{N-1}
$$

Dessa forma, considera-se que $\mu_{1}$ e $\mu_{2}$ correspondem às respectivas médias mensais dos dados reais e sintéticos, $\mathrm{N}_{1}$ e $\mathrm{N}_{2}$ são as amostras mensais analisadas, $\sigma$ o desvio padrão entre as séries e $s_{1}^{2}$ e $s_{2}^{2}$ as respectivas variâncias de cada série. Já quanto ao grau de liberdade da distribuição $t$, adotou-se:

$$
\mathrm{V}=N_{1}+N_{2}-2
$$

Os resultados da estatística $t$ de Student permitiram verificar se os dados estimados pelo TRMM podem ser considerados, estatisticamente, homogêneos às precipitaçóes observadas na estação do INMET de Uberaba.

\section{RESULTADOS}

Nas Tabelas 1 e 2 estão dispostos os totais mensais de precipitação observados na Estação do INMET de Uberaba (Tabela 1) e os totais mensais estimados pelo satélite TRMM para o período de 2000-2012 (Tabela 2), bem como suas estatísticas descritivas. 
Tabela 1 - Totais pluviométricos mensais observados na Estação Meteorológica de Superfície do INMET - Uberaba e estatística descritiva

\begin{tabular}{|c|c|c|c|c|c|c|c|c|c|c|c|c|}
\hline & JAN & FEV & MAR & ABR & MAI & JUN & JUL & AGO & SET & OUT & NOV & DEZ \\
\hline 2000 & 523,1 & 350,0 & 392,6 & 85,2 & 6,2 & 0 & 9,1 & 6,4 & 112,6 & 54,6 & 190,6 & 351,1 \\
\hline 2001 & 175,2 & 146,9 & 173,9 & 24,9 & 27,7 & 0 & 23,9 & 74,1 & 30,0 & 94,0 & 212,4 & 242,7 \\
\hline 2002 & 308,8 & 354,7 & 126,0 & 9,3 & 66,0 & 0 & 13,0 & 2,0 & 53,8 & 71,4 & 190,7 & 206,1 \\
\hline 2003 & 550,3 & 190,6 & 336,9 & 73,0 & 56,3 & 0 & 0 & 12,7 & 50,5 & 91,6 & 154,1 & 221,1 \\
\hline 2004 & 214,4 & 359,8 & 168,6 & 143,0 & 58,2 & 36,6 & 31,4 & 0 & 6,0 & 136,9 & 113,0 & 482,8 \\
\hline 2005 & 395,2 & 110,3 & 258,8 & 32,7 & 103,6 & 0 & 5,5 & 38,6 & 73,7 & 144,2 & 217,6 & 298,2 \\
\hline 2006 & 245,3 & 287,5 & 285,6 & 65,8 & 46,2 & 2 & 0 & 27,5 & 51,9 & 241,2 & 187,5 & 299,7 \\
\hline 2007 & 414,9 & 198,2 & 104,9 & 109,6 & 17,3 & 0 & 15,7 & 0 & 10,2 & 166,5 & 245,8 & 317,6 \\
\hline 2008 & 341,9 & 385,3 & 320,8 & 235,4 & 75,2 & 0 & 0 & 18,8 & 40,2 & 68,8 & 121,0 & 450,3 \\
\hline 2009 & 291,2 & 251,3 & 200,6 & 48,4 & 41,5 & 28,9 & 11,4 & 20,9 & 88,1 & 152,4 & 153,5 & 339,3 \\
\hline 2010 & 411,2 & 169,5 & 211,8 & 96,0 & 18,3 & 9,5 & 0,0 & 0 & 72,1 & 176,4 & 366,9 & 226,3 \\
\hline 2011 & 284,7 & 188,1 & 629,3 & 301,2 & 0 & 25,1 & 0 & 2,9 & 2,0 & 151,6 & 148,8 & 213,7 \\
\hline 2012 & 415,5 & 156,0 & 128,5 & 86,6 & 73,0 & 91,0 & 10,2 & 0 & 30,6 & 35,5 & 221,8 & 169,2 \\
\hline Média & 351,7 & 242,2 & 256,8 & 100,9 & 45,3 & 14,9 & 9,2 & 15,7 & 47,8 & 121,9 & 194,1 & 293,7 \\
\hline Mediana & 341,9 & 198,2 & 211,8 & 85,2 & 46,2 & 0 & 9,1 & 6,4 & 50,5 & 136,9 & 190,6 & 298,2 \\
\hline Máx. & 550,3 & 385,3 & 629,3 & 301,2 & 103,6 & 91,0 & 31,4 & 74,1 & 112,6 & 241,2 & 366,9 & 482,8 \\
\hline Mín. & 175,2 & 110,3 & 104,9 & 9,3 & 0 & 0 & 0 & 0 & 2,0 & 35,5 & 113,0 & 169,2 \\
\hline Amp. & 375,1 & 275,0 & 524,4 & 291,9 & 103,6 & 91,0 & 31,4 & 74,1 & 110,6 & 205,7 & 253,9 & 313,6 \\
\hline D. Pad & 113,2 & 94,6 & 142,9 & 83,7 & 30,5 & 26,3 & 10,0 & 21,5 & 33,0 & 58,3 & 65,5 & 95,0 \\
\hline CV (\%) & 32 & 39 & 56 & 83 & 67 & 177 & 109 & 137 & 69 & 48 & 34 & 32 \\
\hline
\end{tabular}

Legenda: Máx: valor máximo; Mín: valor mínimo; Amp: Amplitude; D.Pad: Desvio padrão da série; CV:

Coeficiente de Variação (\%).

Muito embora se observe que para alguns meses os dados propostos pelo satélite TRMM estão superestimados, em outros meses os dados TRMM mostram-se subestimados. A estatística descritiva aplicada a ambas as séries apresenta valores com forte similaridade.

As diferenças nos valores médios de ambas as séries são muito sutis, assim como os resultados encontrados para seus desvios padróes.

Collischonn et al. (2006) acreditam que a estimativa de precipitação do satélite TRMM é bastante precisa quando comparada com dados de solo. Dessa forma, a estimativa de satélite pode ajudar a identificar pluviômetros com problemas de leitura ou ainda, pluviômetros mal localizados, constituindo-se em ferramenta para consistência de dados. 
Tabela 2 - Totais pluviométricos mensais propostos pelo satélite TRMM para Uberaba e estatística descritiva

\begin{tabular}{|c|c|c|c|c|c|c|c|c|c|c|c|c|}
\hline & JAN & FEV & MAR & ABR & MAI & JUN & JUL & AGO & SET & OUT & NOV & DEZ \\
\hline 2000 & 475,4 & 347,8 & 272,4 & 61,0 & 1,0 & 0 & 9,1 & 8,0 & 146,4 & 67,8 & 216,0 & 258,3 \\
\hline 2001 & 213,0 & 174,7 & 177,1 & 42,3 & 78,1 & 0 & 16,5 & 43,7 & 49,8 & 65,8 & 237,0 & 334,0 \\
\hline 2002 & 259,5 & 357,7 & 115,5 & 11,5 & 51,7 & 0 & 4,3 & 6,0 & 59,5 & 43,0 & 148,2 & 257,1 \\
\hline 2003 & 605,2 & 197,9 & 243,7 & 128,3 & 51,7 & 0 & 3,1 & 13,4 & 69,1 & 53,9 & 251,9 & 172,7 \\
\hline 2004 & 294,2 & 272,8 & 137,3 & 113,6 & 46,0 & 19,4 & 41,8 & 0 & 8,3 & 115,5 & 144,8 & 379,4 \\
\hline 2005 & 364,1 & 102,1 & 205,8 & 61,3 & 100,6 & 37,1 & 4 & 5,2 & 100,8 & 78,0 & 295,9 & 304,7 \\
\hline 2006 & 263,1 & 327,8 & 224,8 & 109,6 & 17,0 & 18,0 & 1,8 & 16,7 & 48,2 & 227,2 & 215,2 & 363,1 \\
\hline 2007 & 473,4 & 213,0 & 97,6 & 81,6 & 20,1 & 0 & 69,1 & 0 & 7,5 & 58,1 & 182,8 & 296,3 \\
\hline 2008 & 306,5 & 316,1 & 311,4 & 177,4 & 31,6 & 16,4 & 0 & 26,1 & 37,4 & 83,9 & 112,0 & 360,4 \\
\hline 2009 & 406,4 & 280,0 & 323,6 & 102,2 & 87,0 & 24,3 & 5,3 & 37,5 & 100,5 & 189,7 & 197,6 & 324,3 \\
\hline 2010 & 292,9 & 232,6 & 239,1 & 77,1 & 24,5 & 8,0 & 0 & 0 & 80,6 & 170,5 & 248,8 & 229,5 \\
\hline 2011 & 406,3 & 179,1 & 478,9 & 154,1 & 2,2 & 18,5 & 0 & 5,1 & 9,5 & 179,7 & 153,9 & 317,5 \\
\hline 2012 & 449,8 & 144,9 & 170,3 & 112,8 & 36,6 & 106,3 & 10,9 & 0 & 43,7 & 67,5 & 217,9 & 239,1 \\
\hline Média & 370,0 & 242,0 & 230,6 & 94,8 & 42,2 & 19,1 & 12,8 & 12,4 & 58,6 & 107,7 & 201,7 & 295,1 \\
\hline Mediana & 364,1 & 232,6 & 224,8 & 102,2 & 36,6 & 16,4 & 4,3 & 6,0 & 49,8 & 78,0 & 215,2 & 304,7 \\
\hline Máx. & 605,2 & 357,7 & 478,9 & 177,4 & 100,6 & 106,3 & 69,1 & 43,7 & 146,4 & 227,2 & 295,9 & 379,4 \\
\hline Mín. & 213,0 & 102,1 & 97,6 & 11,5 & 1,0 & 0 & 0 & 0 & 7,5 & 43,0 & 112,0 & 172,7 \\
\hline Amp. & 392,2 & 255,6 & 381,3 & 165,9 & 99,6 & 106,3 & 69,1 & 43,7 & 138,9 & 184,2 & 183,9 & 206,7 \\
\hline D. Pad & 111,9 & 81,7 & 102,3 & 45,5 & 31,3 & 28,7 & 20,3 & 14,7 & 41,1 & 62,0 & 51,9 & 60,7 \\
\hline CV (\%) & 30 & 34 & 44 & 48 & 74 & 151 & 159 & 118 & 70 & 58 & 26 & 21 \\
\hline
\end{tabular}

Legenda: Máx: valor máximo; Mín: valor mínimo; Amp: Amplitude; D.Pad: Desvio padrão da série; CV:

Coeficiente de Variação (\%).

A Figura 2 demonstra a comparação entre os dados mensais observados com os estimados pelo TRMM utilizando a técnica do Box Plot.

Os gráficos da Figura 2 demonstram que o satélite TRMM subestimou os dados pluviométricos nos meses de fevereiro, março, abril, maio, junho, julho, agosto e dezembro, mantendo-os mais próximos da tendência central (mediana). Especificamente, para os meses de abril e agosto, o TRMM subestimou, também, os eventos pluviométricos intensos, mantendo o conjunto de dados mais próximos da mediana.

Já os dados de janeiro e novembro foram superestimados pelo TRMM. Os gráficos para esses meses na Figura 2 demonstram aumento na dispersão dos dados quando comparados aos observados na estação do INMET. Para o mês de outubro, o TRMM subestimou o conjunto de dados, sugerindo redução no valor da mediana. 
Figura 2 - BoxPlot dos totais mensais de precipitação observados (INMET) e estimados pelo TRMM

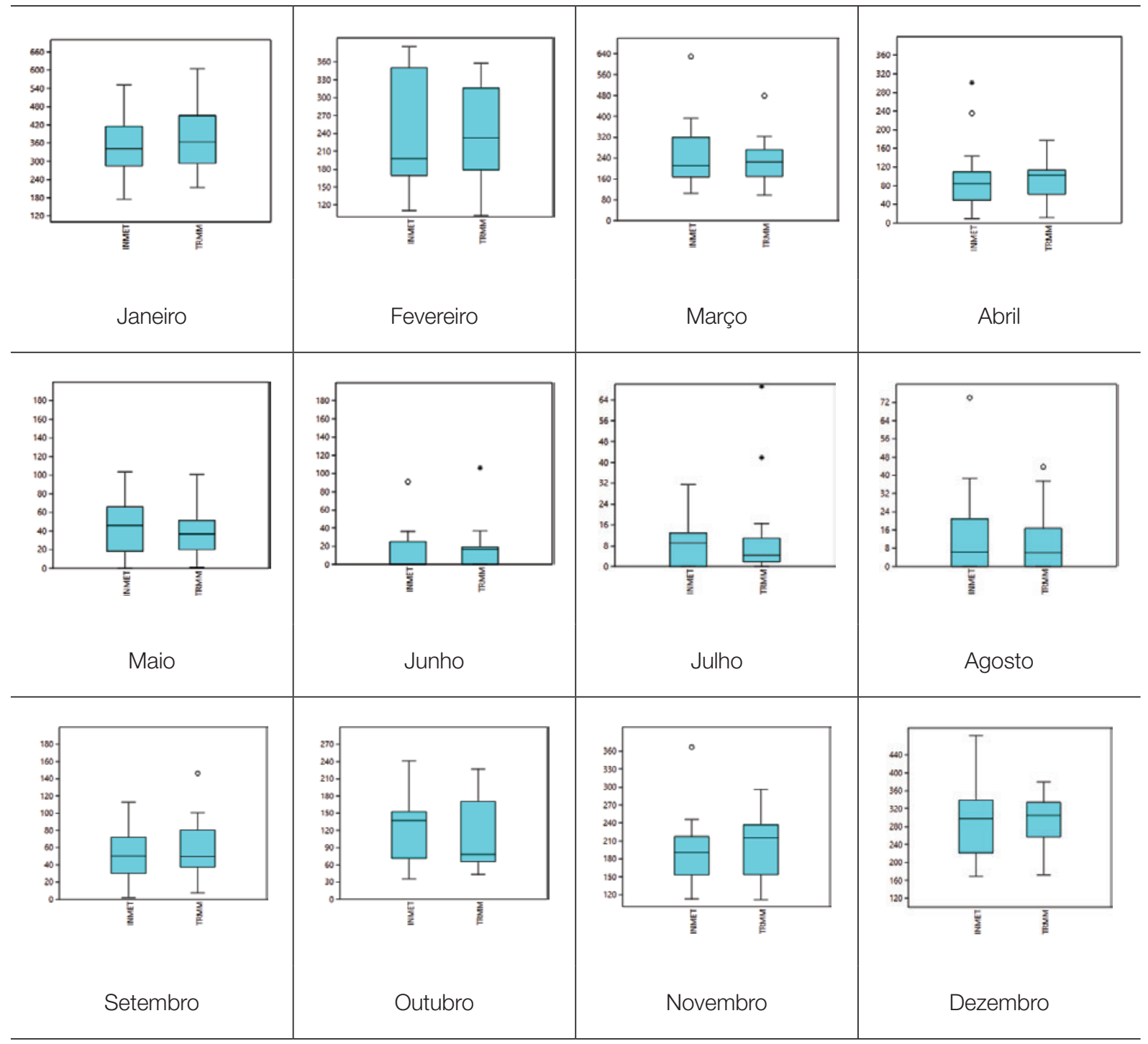

Fonte: Dados analisados do INMET e do INPE/TRMM

A aplicaçáo da Correlação de Pearson entre os dados observados (INMET) e os dados estimados (TRMM)(Figura 3) revelou o elevado grau de correlaçáo uma vez que a maior parte de ambos os conjuntos de dados posicionou-se, praticamente, sobre a reta de regressão, bem como o elevado coeficiente de determinação $\left(\mathrm{R}^{2}=0,994\right)$ entre ambas as séries. 
Figura 3 - Correlação entre os dados observados na Estação Meteorológica do INMET e os dados estimados pelo satélite TRMM para Uberaba (MG)

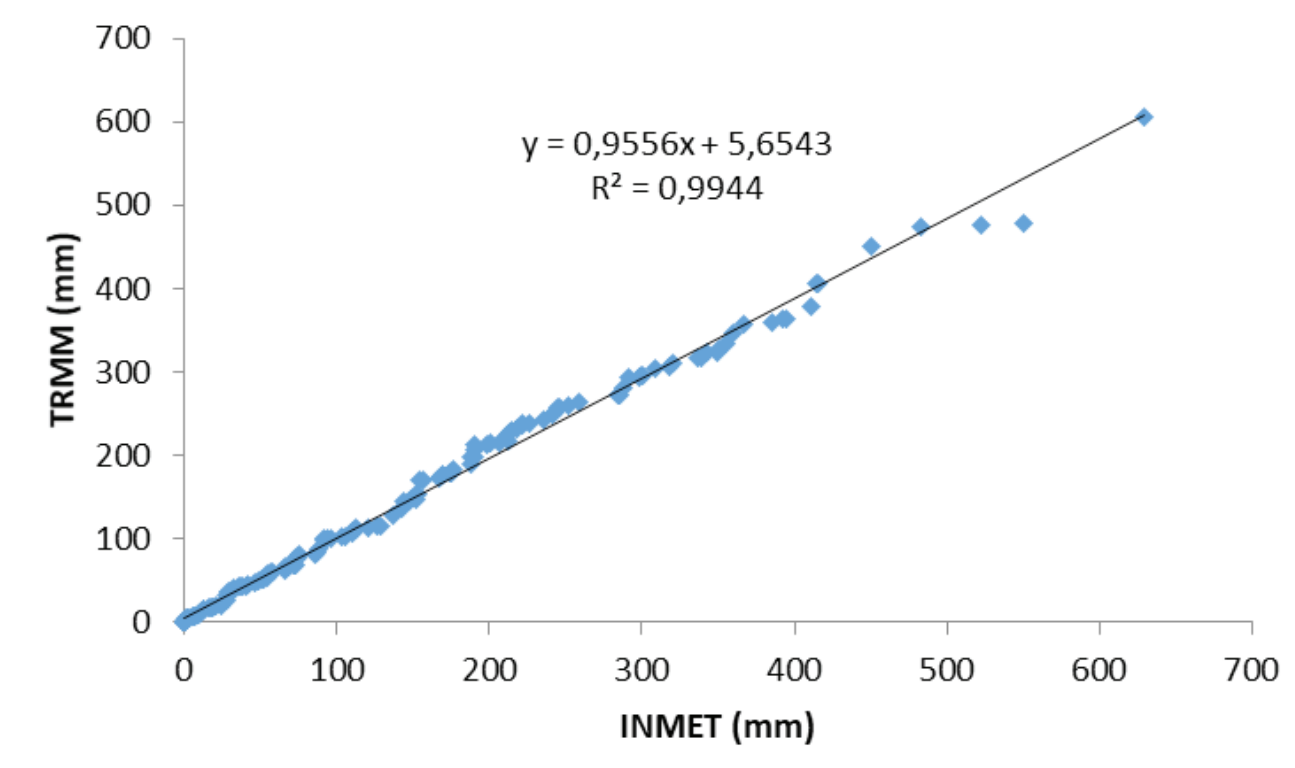

Fonte: Dados analisados do INMET e do INPE/TRMM

Por fim, a Tabela 3 mostra o resultado da aplicação do Teste $t$ de Student para a verificação da homogeneidade entre os dados de ambos os sítios utilizados. Considerando o nível de significância de $95 \%(p$-value $=0,05)$ a hipótese $H_{0}$ (de que ambas as séries são, estatisticamente, homogêneas) deverá ser rejeitada sempre que o valor " $z$ " encontrado estiver $-1,96<\mathrm{z}<+1,96$ (SPIEGEL, 1993).

Tabela 3 - Estatística (z) do Teste $t$ de Student para os dados observados na Estação do INMET e propostos pelo satélite TRMM para Uberaba, no período 2000-2012.

\begin{tabular}{c|c|c|c|c|c|c|c|c|c|c|c}
\hline Jan. & Fev. & Mar. & Abr. & Maio & Jun. & Jul. & Ago. & Set. & Out. & Nov. & Dez. \\
\hline$-0,41$ & 0,00 & 0,54 & 0,23 & 0,27 & $-0,39$ & $-0,55$ & 0,45 & $-0,73$ & 0,60 & $-0,33$ & $-0,05$ \\
\hline
\end{tabular}

Fonte: organizado pelos autores a partir da aplicação do

Teste t de Student aos dados do INMET e INPE/TRMM.

Os resultados da estatistica (z) do Teste $t$ de Student demonstram que a hipótese $H_{0}$ pode ser aceita para todos os meses, considerando que os dados de ambas as séries são, estatisticamente, homogêneos.

Dessa forma, corroborando com os resultados encontrados em Collischon et al. (2006; 2007), Nobrega et al. (2008) e Anjos et al. (2016), é possível utilizar dados obtidos do satélite TRMM para Uberaba uma vez que eles representam, muito bem, o comportamento das chuvas mensais, sendo uma alternativa para as possíveis ausências de dados em suas séries temporais. 


\section{CONCLUSÓES}

Com base nos dados utilizados e na metodologia proposta, é possível considerar que a aplicação da estatística descritiva revelou forte similaridade entre os dados observados na estação meteorológica de superfície para Uberaba e os estimados pelo satélite TRMM para o mesmo local.

A avaliação feita pela técnica do Box Plot demonstrou que na maior parte dos meses os dados do TRMM subestimaram os valores mensais de pluviosidade, sobretudo em relação aos valores pluviométricos intensos. Verificou-se, também, que a tendência central dos dados TRMM permaneceu semelhante aos dados observados.

A aplicação da Correlação de Pearson apresentou um elevado nível de correlação, principalmente pelo seu excelente coeficiente de determinação $\left(\mathrm{R}^{2}=0,994\right)$.

Por fim, a aplicação da estatística $t$ de Student considerou que ambos os conjuntos de dados são homogêneos, ou seja, de que não há diferenças significativas entre os conjuntos de dados analisados.

Dessa forma, a partir da metodologia proposta, os resultados demonstraram que os dados do satélite TRMM possuem excelente qualidade quando comparados aos dados observados em superfície para Uberaba (MG). 


\section{REFERÊNCIAS BIBLIOGRÁFICAS}

ANJOS, R.; CANDEIAS, A.L.B.; NÓBREGA, R.S. Caracterização das chuvas através do satélite TRMM em Petrolândia (PE). Revista Brasileira de Climatologia. v.18, jan.- jun., 2016. Disponível em:<http://revistas.ufpr.br/revistaabclima/article/view/44652>. Acesso em: 31 out. 2016.

COLLISCHONN, B. Uso de precipitação estimada pelo satélite TRMM em modelo hidrológico distribuído. 2006. 128 f. Dissertação. (Mestrado em Recursos Hídricos e Saneamento Ambiental) Universidade Federal do Rio Grande do Sul, Porto Alegre,2008.

. et al. Desempenho do satélite TRMM na estimativa de precipitaçáo sobre a bacia do Paraguai superior. Revista Brasileira de Cartografia, Porto Alegre, v.59, n.1, pp. 93-99, 2007.

COLLISHONN, B.; COLLISHONN, W; TUCCI, C. Análise do campo de precipitação gerado pelo satélite TRMM sobre a bacia do São Francisco até Três Marias. In: SIMPÓSIO DE RECURSOS HÍDRICOS DO SUL, . Anais..., Curitiba, 2006.

DA SILVA, D.F.; PRELA-PANTANO, A.; CAMARGO, M.B. P. Análise de dados de precipitação estimados pelo satélite TRMM para o Vale do Médio Parapanema (SP). REVENG - Engenharia na agricultura, Viçosa (MG), v.21 n.2, mar.-abr. 2013. Disponível em:<http://dx.doi.org/10.13083/1414-3984.v21n02a04>. Acesso em:

NOBREGA, R.; SOUZA, E. P. GALVÍNIO, J.D. Análise da estimativa de precipitação do TRMM em uma sub-bacia da amazônia ocidental. Revista de Geografia, Recife: UFPE DCG/NAPA, v. 25, n. 1, jan.-abr. 2008.

SANCHES, F. O. O Geógrafo-Climatologista e as mudanças climáticas: uma proposta metodológica. Revista Equador, v. 4, pp. 101-118, 2015.

SPIEGEL, M. R. Estatística. Tradução e revisão: CONSENTINO, p. 3.ed. São Paulo: Pearson Makron Books/McGraw-Hill do Brasil, 1993.

Recebido para publicação em Agosto de 2017 Aceito para publicaçâo em Dezembro de 2017 